\title{
A variable mapping task produces symmetrical interference between global information and local information
}

\author{
JUDITH M. SHEDDEN and GRAEME S. REID \\ McMaster University, Hamilton, Ontario, Canada
}

\begin{abstract}
When processing global and local aspects of compound visual figures, a robust finding is that global targets are detected faster and more accurately than local targets. Moreover, unidirectional interference is often observed. Despite the convincing evidence that global information and local information are available together, when attention is focused on the global level, items from the local level often have very little, if any, effect on behavior. If local information is available with global information, then why is global dominance so often observed under such a wide range of conditions? This paper is concerned with the fate of the ignored, and apparently ineffective, local distractors. In our experiments, at least one critical factor was stimulus-response (S-R) mapping. We compared a consistent S-R task, which facilitated a speed advantage for global, with a variable $S-R$ task, which required a higher degree of semantic analysis for each stimulus. The two tasks produced large differences in behavior, showing unidirectional interference in the consistent S-R task, and strong bidirectional interference in the variable $\mathrm{S}-\mathrm{R}$ task. Thus, the identity of ignored local distractors was available, even under conditions that favored focused attention to global information. The results provide support for a model in which global processing proceeds more quickly at early perceptual stages and in which local processing can catch up if processing demands are increased at later stages.
\end{abstract}

When processing global and local aspects of compound visual figures, global dominance over local processing is a remarkably robust phenomenon. Much research has been devoted to the investigation of the locus and the mechanisms involved; however, there is still little consensus with respect to the interpretation of the effect. The phenomenon can be described as follows. Under a wide variety of stimulus and task manipulations, it is often observed that responses to global targets are faster than responses to local targets. Moreover, there is asymmetric interference: Global distractors affect local responses, but not vice versa (Briand, 1993, 1994; Hoffman, 1980; Hughes, Layton, Baird, \& Lester, 1984; Navon, 1977, 1981; Paquet, 1992; Paquet \& Merikle, 1988). One interpretation of the results is that the Gestalt configuration or global form leads processing, followed by an analysis of the local detail, a phenomenon that has come to be known as global precedence (Navon, 1977). Even though a number of studies suggest that global information and local information are available together (e.g., Boer \& Keuss, 1982; Hoeger, 1997; Miller, 1981), global processing ap-

This research was supported by Natural Sciences and Engineering Research Council of Canada Grant OGP0170353 to J.M.S. Thanks to Lise Paquet, Kevin Briand, Lee Brooks, Colin M. MacLeod, and an anonymous reviewer for constructive criticism of an earlier draft of this manuscript. Thanks to Stephanie Hevenor for assistance collecting data. Correspondence should be addressed to J. M. Shedden, Department of Psychology, McMaster University, Hamilton, ON L8S 4K1, Canada (e-mail: shedden@mcmaster.ca). pears to dominate local processing (see Kimchi, 1992, for a review). The puzzle that still remains to be solved surrounds the mechanism by which global dominance occurs: We still do not understand the source of the global advantage.

\section{When Do Local Elements Influence Global Processing?}

There are many studies that show a reversal of the global advantage, so that local elements show a processing advantage over global elements, or at least influence global responses. However, such a reversal is most often due to stimulus manipulations that alter the perceptual discrimination of one hierarchical level over another. For example, studies have altered perceptual discrimination by manipulating factors, such as eccentricity and stimulus position uncertainty (Grice, Canham, \& Boroughs, 1983), stimulus distortion (Hoffman, 1980), visual angle and size ratio of global/local elements (Kinchla \& Wolfe, 1979), peripheral presentation (Lamb \& Robertson, 1988), local element density (Klein \& Barresi, 1985; Martin, 1979), spatial frequency (Hughes, Fendrich, \& Reuter-Lorenz, 1990), and stimulus exposure duration (Paquet $\&$ Merikle, 1984).

For example, Grice et al. (1983) showed that global dominance was eliminated when fixation was controlled to ensure that the local elements were adequately perceived. Likewise, if the density of local elements is reduced, the global item becomes more difficult to perceive, and this results in local dominance (Martin, 1979). The direction of the advantage can also be reversed by 
increasing the size of the hierarchical figure. For example, when the global figure is very large (greater than $8^{\circ}$ of visual angle), local dominance is observed (Kinchla \& Wolfe, 1979). Moreover, the transition from a global processing advantage to a local advantage depends on the total set or range of visual angles used (Lamb \& Robertson, 1990). These and other studies support the idea that the perceptual discriminability of the information at both levels strongly influences whether a global or local processing advantage occurs (Kimchi, 1992). Interference between the two levels is relative, depending on the quality of both global information and local information. In other words, the level for which the items are most discriminable shows processing dominance (Hoffman, 1980; Pomerantz, 1983).

However, the global advantage is not a function of perceptual discriminability alone. In fact, even when the global and local elements are equated for baseline perceptual discriminability (values along one dimension are as different perceptually and psychologically as values along the other dimension; Melara \& Mounts, 1993), a global processing advantage is most often observed.

\section{When Local Information Appears to be Unavailable}

Unless the perceptual goodness of the global level relative to the local level is compromised, it is difficult to observe local-to-global interference, even when attention is first directed to the local level. For example, $\mathrm{Pa}$ quet and Merikle (1988) presented two global/local figures, one to the left and one to the right of fixation. One of the compound figures was framed in a square, and the other was framed in a circle. Participants were informed ahead of time whether to attend to the global level or the local level of the figure embedded in the square or the circle. Distractors were congruent, incongruent, or neutral with respect to the target response. Within the attended object, global dominance was observed; global targets were identified more quickly than local targets, and there was unidirectional interference (incongruent global distractors delayed responses to local targets, but there was no effect of congruency due to local distractors). To eliminate the possibility that the location cue (square or circular frame) acted to bias participants to first process the global information, Paquet (1992) used local information as a cue for selection of the relevant object. A small gap in the square or circular frame indicated the relevant object. Even with this local cue, unidirectional globalto-local interference was observed, providing evidence for the priority of global processing.

Why does the local information appear to be unavailable in these experiments? This is especially puzzling given the evidence that both global information and local information are available at the same time (Boer \& Keuss, 1982; Hoeger, 1997; Miller, 1981). One possible explanation is that, under conditions of global attention, the ignored local information is efficiently filtered at a relatively early stage by default and is not consulted under any circumstances until global information has reached some minimum stage of processing. In the case of a default filter, practice should not affect the results. However, Paquet (1992) demonstrated convincingly that this was not the case by giving observers five sessions of extended local practice, after which response times (RTs) were equal for local and global targets, and bidirectional interference was obtained. Thus, extended local practice reduced the efficiency of the global filter, clearly demonstrating that the filter is not a default mechanism (Paquet, 1992).

If local information is not filtered by default, then another possibility is that the filter is strategic, with the ability to efficiently filter local information when it is known to be irrelevant. For example, Miller(1981) showed convincingly that information about both global and local elements is available at the same time (see also Boer \& Keuss, 1982; Hoeger, 1997; Hübner, 1997; Parker, Lishman, \& Hughes, 1996). When attention was divided across levels and when targets appeared at both levels, responses were faster than when the target appeared at either the global or the local level alone, indicating that information from both levels was integrated. In other words, it was not the case that the dominant level (global) completed first and determined the response (as in a race model in which the response is determined by the faster decision to be made); otherwise, there would be no difference between the condition in which there was a single global target and the condition in which there were two targets, one global and one local. Rather, because the twotarget condition was significantly faster than the fastest condition in the single-target case, the overall decision must have been made before a decision could be made about either source alone. In Miller's terms, when attending to both levels, information is integrated by a single decision process.

Importantly, only when attention was divided did local items affect global processing; when attention was focused on global or local items, unidirectional global-tolocal interference was obtained. Miller (1981) argued that global dominance is not an issue of order of availability but a difference in the ease of directing attention. In Miller's model, global information and local information feed into the same decision process at the same time; however, it is easier to attend to and base decisions on the global information because it is more salient or attention grabbing. Moreover, the global and local channels are not completely separable. Therefore, enhancement or attenuation of the signal due to attention would affect both channels. Miller claimed that the global channel has greater strength than the local channel and that it is possible to attend to global information and entirely filter local information, but not the other way around (Miller, 1981).

In a series of experiments examining interference from interobject incongruent distractors, Briand $(1993,1994)$ explored the attentional filter hypothesis by making the following prediction: If the unidirectionalglobal-to-local interference is due to an attentional mechanism that acts as an efficient filter at an early stage, then compromising the attentional mechanism should compromise the effi- 
ciency of the local filter. In other words, local-to-global interference should be observed. Briand's design was similar to Paquet's (1992) in the sense that attention was directed to one of two simultaneously presented compound figures, and interference was measured from incongruent distractors present in the unattended figure (interobject interference). In contrast to Paquet, and similar to Miller's (1981) divided attention experiment, Briand presented mixed trials during which participants did not know from trial to trial whether the target would appear at the global level or the local level; thus, attention was divided across global and local levels. First, he showed that performance was indeed compromised in the divided attention condition, in terms of increased RTs and errors, indicating that response selection was more difficult. Second, incongruent distractors in the unattended location interfered with responses to targets only if they were at the same global or local level, consistent with the results of Paquet and Merikle (Paquet, 1992; Paquet \& Merikle, 1988). Moreover, there was a category effect showing unidirectional global-to-local interference, such that global distractors (irrespective of congruency) interfered with responses to local targets, but not vice versa. In other words, compromising the attentional filter did not appear to compromise the apparent efficiency of the global filter, at least in terms of interobject interference.

Briand's (1993) divided attention results provide strong evidence that interference must occur later in processing. Local interference was not observed even though participants did not know the level at which the target would occur and thus could not select the correct level without some processing of items at both levels. As Briand (1993) pointed out, his results are consistent with models of unlimited capacity in which identification occurs for all stimuli, but distractors can be effectively ignored given appropriate selection cues (Duncan, 1981) or if the ignored information decays before interference occurs (Van der Heijden, 1981). Importantly, identification may occur even under conditions for which there is no effect on behavior. The lack of effect on behavior is not necessarily indicative of the stage to which unattended information is processed (Duncan, 1981). On the basis of these results, Briand suggested that, in principle, it should be possible to show that ignored local items are identified; however, he was not able to do so with his procedure (Briand, 1993).

The question remains, then, with respect to why local information appears to be unavailable. There is sufficient evidence that global information and local information are available simultaneously (Boer \& Keuss, 1982; Briand, 1993; Evans, Shedden, Hevenor, \& Hahn, 2000; Miller, 1981). Yet there was no interobject local interference in a divided attention task even though the selection process was compromised (Briand, 1993), and there was no intraobject interference in a focused attention task even though the stimulus remained on the screen until the response was made (Miller, 1981), a pattern consistent with other studies of global/local interference (Paquet, 1992; Paquet \& Merikle, 1988).

Note the similarity between Briand's (1993) and Miller's (1981) arguments. Both argue for a late selection process that is affected by a greater efficiency of processing for global elements. Perhaps global precedence arises at least in part from the more efficient processing of lower spatial frequencies in the magnocellular stream (Breitmeyer, 1975; De Valois \& De Valois, 1988; Hughes, Nozawa, \& Kitterle, 1996), resulting in greater salience and attentiongrabbing power because of faster conduction of information at early stages of processing. This does not preclude the argument that local information and global information accumulate at the same time; it simply suggests that global information accumulates faster at the earliest stages and that attention may amplify this difference (Hübner, 1997).

Attention leads to a further advantage for global processing if the task allows a fast response. For example, suppose global information accumulates enough to trigger a response. Local information is accumulating as well but does not reach a stage where the information is sufficient to interfere with the global response. However, the advantage for global processing should be reduced if fast responses are not possible. A similar hypothesis was suggested by Hübner (1997, p. 199), who noted that when fast processing is not possible, there is a decrease in the contribution of low spatial frequencies to the global advantage.

Imagine that the response mapping is made more difficult so that when enough information has accumulated, a complex response must be determined. If this increases the time needed at later stages, the total RT will have less of a contribution from the early stages. Then, even under global attention conditions, before response selection has completed, enough local information may have accumulated to begin to interfere with the response. The same argument applies to local attention, except that the processing delay will not be as great because local processing is affected by the stronger global channel in any case. In other words, the interference from global items during local attention is not an additional factor, whereas the interference from local items during global attention is an additional factor.

How might we change the response mapping to make the task more difficult at later stages? Almost invariably, global/local experiments of the type discussed above have used small target sets. In addition, to facilitate testing of congruent and incongruent distractor types, studies most often use a consistent mapping between target sets and responses. That is, over the course of a block of trials, observers search for a particular target associated with a consistent response among a consistent set of distractors.

However, it may be that the global processing speed advantage is facilitated by the consistent stimulusresponse (S-R) mapping design used in most global/local experiments. Training with a set of consistently mapped stimuli has been shown to lead to increases in the efficiency of responding to those stimuli (Logan \& Stadler, 
1991; Schneider \& Shiffrin, 1977; Shiffrin \& Schneider, 1977). Interference that occurs at the response stage in a consistently mapped task may preclude interference at intermediate stages of processing if automatically elicited responses bypass or speed intermediate stages. For example, once an automatic response to a stimulus has developed, and when the stimulus set is small, very little perceptual information may be necessary to trigger the response. Intermediate steps related to stimulus evaluation, which may be critical in terms of interference between competing sources of information, may not contribute as much once an automatic response has developed. Thus, stimuli that are consistently mapped to the same response elicit faster responses, leading to interference that occurs in only one direction (global to local) because of the inherently faster processing of global information at early stages.

In that case, a variable S-R mapping paradigm, which demands a more thorough evaluation of each stimulus on each trial, should make the later stages of processing more difficult. Forcing a more complete evaluation of each stimulus will affect the later processes that occur between perception and response. These are the same intermediate stages of processing that are bypassed or speeded when automaticity develops (Schneider \& Shiffrin, 1977). The prediction is that we should observe bidirectional interference, even when attention is focused on global or local levels.

In this paper, we present a novel variable $S-R$ task with certain critical elements that are different from the typical designs used to test global/local processing. In Experiment 1, we used a relatively large stimulus set (e.g., 99 hierarchical stimuli). More importantly, stimuli and responses were variably mapped across and within blocks of trials: Any particular stimulus was sometimes a target and sometimes a distractor. In other words, the items that made up the target and distractor sets changed on every trial. The result was that detection of any particular stimulus did not automatically trigger a specific response, but evaluation of each stimulus had to be carried out to completion in a controlled manner. In contrast, in Experiment 2 , we used a consistent S-R mapping. In both experiments, we manipulated stimulus congruency by presenting information at the ignored level that was congruent or incongruent with the information at the attended level. On the incongruent trials, we demonstrated a dramatic difference in behavior between the variable and consistent $\mathrm{S}-\mathrm{R}$ tasks that may be related to the level of semantic processing required in the variable $S-R$ task versus the automatic and fast responses that develop in the consistent $\mathrm{S}-\mathrm{R}$ task.

\section{EXPERIMENT 1 Variable S-R Mapping}

The number-sequence task involved a variable mapping between stimuli and responses. Attention was not divided but was directed globally or locally for an entire block of trials. The task was a go/no-go task; the participants mon- itored a repeating sequence of digits ( 1 through 9 , repeated three times) and responded to occasional targets defined as any out-of-sequence digit occurring at the attended position and level. For example, in the attended digit sequence " 123956789173456789 ," the first "9" and the second "7" are targets. Note that, depending on its position in the sequence, any particular digit can be a target for a response or a nontarget for which the response must be withheld. Thus, no consistent mapping between a particular stimulus and a response can be made.

On nontarget trials, the distractors presented at the ignored level were out-of-sequence digits. On target trials, the three types of distractors that occurred at the ignored level were congruent, incongruent, or neutral with respect to the target response. For illustration (refer to the example for global attention in Figure 1), consider the fourth trial in a sequence of digits, on which the out-ofsequence global target "9" occurs instead of the expected global "4." The examples in Figure 1 illustrate the three possible types of distractors that could occur at the ignored level when the attended level contained a target. For example, the distractor digit " 7 " is congruent with the response to the target because it is also an out-of-sequence digit; if it appeared at the attended level, it would lead to the execution of the target response (e.g., "go"). The distractor digit " 4 " is the in-sequence digit, which is incongruent with the response to the target; if it appeared at the attended level, it would result in withholding the target response (e.g., "no-go"). Finally, a distractor box, which is never part of the target set, is neutral with respect to the target response.

One prediction is that when attention is directed locally, incongruent global distractors should slow responses to local targets. This prediction is obvious because of the robustness of the global dominance effect.

Two opposing predictions can be made with regard to globally directed attention. The first prediction is that there will be no effect of congruency of local informationthat is, responses to global targets will not depend on the identity of the local digit distractors. This prediction may also seem obvious because we have set up the conditions to favor directed attention. (1) Participants know ahead of time whether to attend to global or local items, and the level of attention is blocked, which are conditions that promote unidirectional global-to-local interference (Briand, 1993; Miller, 1981). (2) Participants are not given extra local practice, which has been shown to lead to bidirectional interference (Paquet, 1992). (3) The distractors on nontarget trials are always out of sequence. Given that out-of-sequence digits at the attended level were targets and required a response, out-of-sequence digits at the ignored level on nontarget trials should have provided strong motivation to select the appropriate level and filter the unattended level, if such filtering is possible as suggested by other studies (Miller, 1981). We might expect that this set of conditions would facilitate the efficiency of globally directed attention and thus produce no local interference effect. 

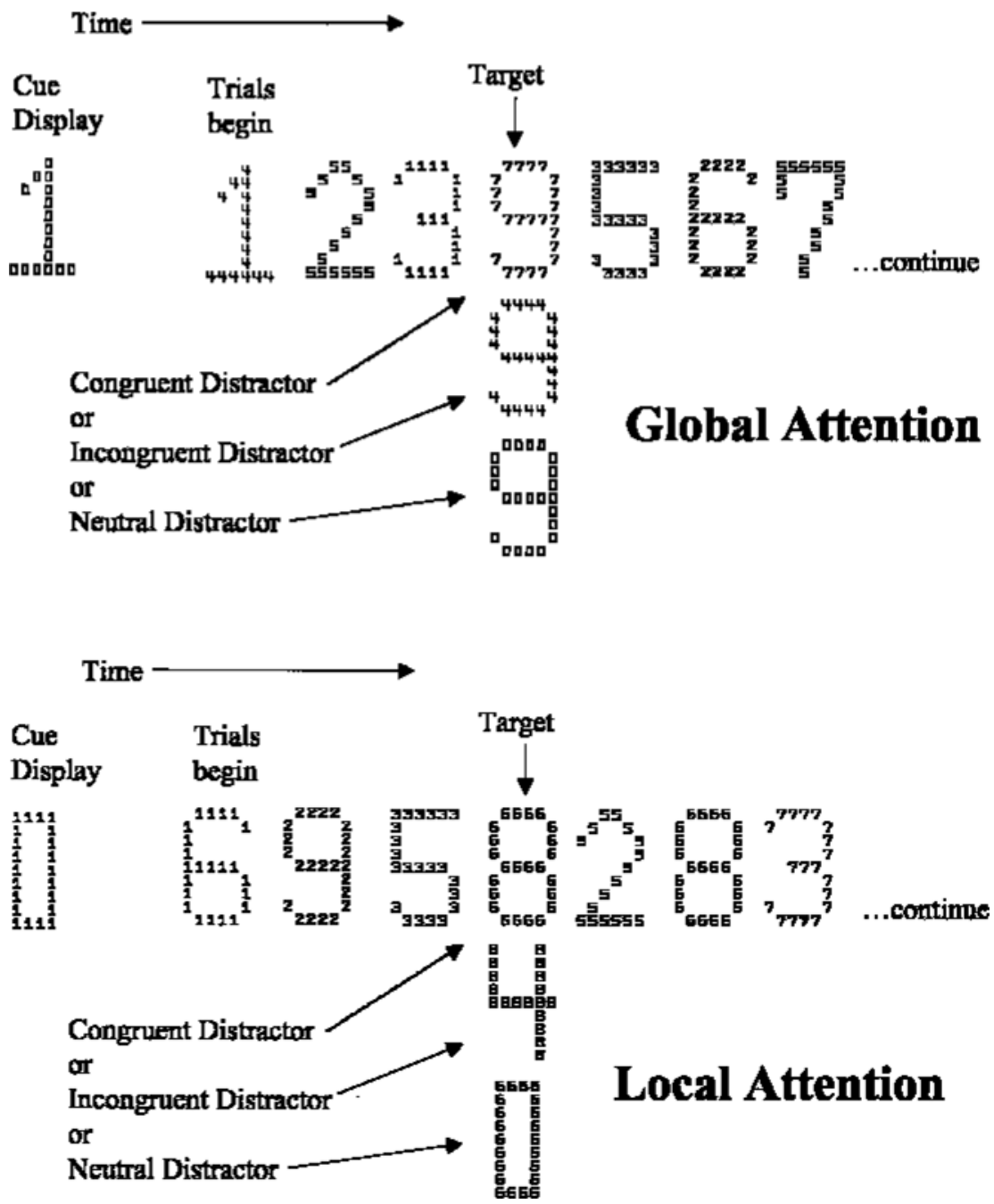

Figure 1. Variable S-R mapping task. Examples of possible sequences of stimuli in the number-sequence task are shown for global attention (upper example) and local attention (lower example). Only the first 7 of 27 trials are shown. The cue display at the beginning of the block of trials informed the participant whether to direct attention globally or locally, and it remained on the screen until the space bar was pressed to start the trials. The onset of the first stimulus occurred after a 1,000-msec delay. Stimulus duration was $100 \mathrm{msec}$, and SOA was $900 \mathrm{msec}$. The participants monitored an ascending sequence of digits, responding to occasional targets (out-of-sequence digits). On target trials, the item at the ignored level was a congruent distractor (the digit at the ignored level was out of sequence, congruent with the target response), an incongruent distractor (the digit at the ignored level was the expected insequence digit, incongruent with the target response), or a neutral distractor (the item at the ignored level was a neutral box, which never required a response).

The less obvious and opposing prediction is that when attention is directed globally, incongruent local distractors will slow responses to global targets. This prediction is based on the hypothesis that global and local features are available together and that ignored local information is processed to the level of identification (Briand, 1993; Duncan, 1981; Van der Heijden, Hagenaar, \& Bloem, 1984). Our task, which requires a more controlled and complete analysis of each attended stimulus, may allow the observation of bidirectional interference under focused attention conditions by increasing processing de- mands at later stages, allowing the ignored local distractors to "catch up" and affect global processing.

\section{Method}

Participants. The participants were 10 undergraduate and graduate students at McMaster University who volunteered to participate either for class credit (undergraduates) or for a small remuneration (graduates). All participants had normal or corrected-to-n ormal vision.

Stimuli. The hierarchical stimuli were global digits (1 through 9) constructed of local digits (1 through 9), producing 81 compound figures, as well as global digits ( 1 through 9) constructed of neutral boxes, and neutral boxes constructed of local digits (1 through 9), 
producing another 18 compound figures. A subset of the 99 hierarchical digit and box figures appears in the examples shown in Figure 1 (details about these example trials are provided below). The boxes were neutral in the sense that they were never associated with a response. The stimuli were constructed by designing the global and local patterns separately and then replacing each pixel in the global pattern with a local pattern. The global digits subtended $1.84^{\circ} \times$ $2.45^{\circ}$ of visual angle (width $\times$ height), and the local digits subtended $0.16^{\circ} \times 0.25^{\circ}$ of visual angle.

Procedure. The participants were seated in front of a computer monitor and made their responses on a computer keyboard. All trials consisted of a single global/local figure presented at fixation. Each block consisted of 27 trials, and attention was directed globally or locally for the entire block. The participants were informed prior to each block whether global or local attention was required. Each participant took part in one hour-long session, and each session consisted of two demonstration blocks (one local and one global), followed by four practice blocks (two local and two global), followed by 60 test blocks in random order ( 30 local and 30 global). The participants were monitored closely during the practice blocks to ensure that they understood the directions and were performing the task correctly. They were instructed to respond as quickly as possible without sacrif icing accuracy.

The independent variables were attended level (global/local), and distractor type (congruent /incongruent /neutral). Attended level was a blocked variable, whereas distractor type was random across trials within each block.

At the beginning of each block, the participants were informed whether to attend to the global level or the local level. This information was presented in the form of a compound figure consisting of "1"s and boxes, with " 1 "s in the to-be-attended level (examples are shown in Figure 1). The cue display remained on the screen until the participant pressed the space bar to initiate the block. A blank screen was then displayed for $1 \mathrm{sec}$, followed by the onset of the first stimulus. Stimulus duration was $100 \mathrm{msec}$, and stimulus onset asynchrony (SOA) was $900 \mathrm{msec}$.

On successive trials, at the attended level (global or local), digits appeared in numerical sequence 1 through 9 . The participants monitored the sequence of digits for occasional targets, which were outof-sequence digits. The participants responded to each target with a keypress. The examples in Figure 1 show the cue display, which indicated whether to attend global or local, followed by possible sequences of global/local figures for the first 7 trials in a block. The fourth trial in each example represents an example target and also shows the three possible types of distractors. Responses were not required to in-sequence digits, and, on these nontarget trials, the distractors at the ignored level were always out-of-sequence digits.

The distractor type manipulation, which refers only to target trials, was implemented as follows. On target trials, the identity of the distractor at the ignored level (i.e., local if attending global; global if attending local) was congruent, incongruent, or neutral with respect to the target (Figure 1). A neutral distractor was a box. A congruent distractor was an out-of-sequence digit (congruent because the target was also an out-of-sequence digit). An incongruent distractor involved the expected in-sequence digit, but it occurred at the unattended level instead of the attended level. Over the course of each block of 27 trials, the probability of a target was approximately .25 , resulting in approximately 6 to 7 targets per block. The probability of each of the three distractor types given a target was .33.

Controls were implemented such that two simultaneous (same trial) or successive (consecutive trials) digits were never the same (including distractor digits, attended digits, or target digits), there were never targets presented in the first two or last two trials, and there were never two consecutive targets. Feedback was provided at the end of each block and consisted of number of hits, misses, and false alarms, as well as the average RT for hits. The participants were given as much time as they wanted to rest between blocks, during which time the cue for the upcoming block was displayed. When ready, the participant initiated the next block by pressing the space bar.

\section{Results}

RTs for Experiment 1 are plotted in the left panel of Figure 2, and means for RT and proportion correct are presented in Table 1.

Response time. Bidirectional interference was observed, such that incongruent distractors at the ignored level slowed both local and global responses. This was tested using a two-factor repeated measures analysis of variance (ANOVA) to examine level (global/local) $\times$ distractor type (congruent/incongruent/neutral). A main effect of level showed that global responses were faster overall than local responses $\left[F(1,9)=5.45, M S_{\mathrm{e}}=2,777\right.$, $p<.05]$. A main effect of distractor type was also observed $\left[F(2,18)=8.76, M S_{\mathrm{e}}=2,083, p<.01\right]$. The interaction between level and distractor type was not significant $\left[F(2,18)=0.4, M S_{\mathrm{e}}=363, p=.68\right]$.

A Scheffé test for post hoc comparison of means examined in more detail the effect of distractor type for global responses and for local responses. Responses to global targets were slower when ignored local distractors were incongruent than when they were congruent $(p<$ $.05)$ or neutral $(p<.001)$; the difference between congruent and neutral local distractors was not significant $(p>.2)$. The same pattern occurred for local attention. Responses to local targets were slower when ignored global distractors were incongruent than when they were congruent $(p<.05)$ or neutral $(p<.001)$; the difference in local target RT between congruent and neutral global distractors was not significant $(p>.1)$.

Accuracy. The effect of local information on global processing was further demonstrated by accuracy. Responses to global targets were less accurate when local distractors were incongruent, and there were no other effects on accuracy. A two-factor repeated measures ANOVA looked at level $\times$ distractor type for proportion correct. The only significant effect was a main effect for distractor type $\left[F(2,18)=11.15, M S_{\mathrm{e}}<0.001, p<.001\right]$. The effect of level $\left[F(1,9)=0.4, M S_{\mathrm{e}}=0.001, p>.5\right]$ and the interaction between level and distractor type $[F(2,18)=3$, $M S_{\mathrm{e}}<.001, p>$.7] were not significant. A further analysis of means (Scheffé test) to examine the effect of dis-

Table 1

Means for Response Times (RTs, in Milliseconds) and for Accuracy (Proportion Correct Targets) in Experiments 1 and 2

\begin{tabular}{|c|c|c|c|c|}
\hline \multirow[b]{2}{*}{ Distractor } & \multicolumn{2}{|c|}{$\begin{array}{l}\text { Experiment 1: } \\
\text { Variable S-R Mapping }\end{array}$} & \multicolumn{2}{|c|}{$\begin{array}{c}\text { Experiment 2: } \\
\text { Consistent S-R Mapping }\end{array}$} \\
\hline & RT & Accuracy & RT & Accuracy \\
\hline \multicolumn{5}{|c|}{ Global Targets } \\
\hline Neutral & 535 & .987 & 407 & .967 \\
\hline Congruent & 558 & .970 & 400 & .972 \\
\hline Incongruent & 590 & .938 & 404 & .958 \\
\hline \multicolumn{5}{|c|}{ Local Targets } \\
\hline Neutral & 561 & .972 & 511 & .963 \\
\hline Congruent & 589 & .968 & 468 & .976 \\
\hline Incongruent & 627 & .932 & 532 & .880 \\
\hline
\end{tabular}




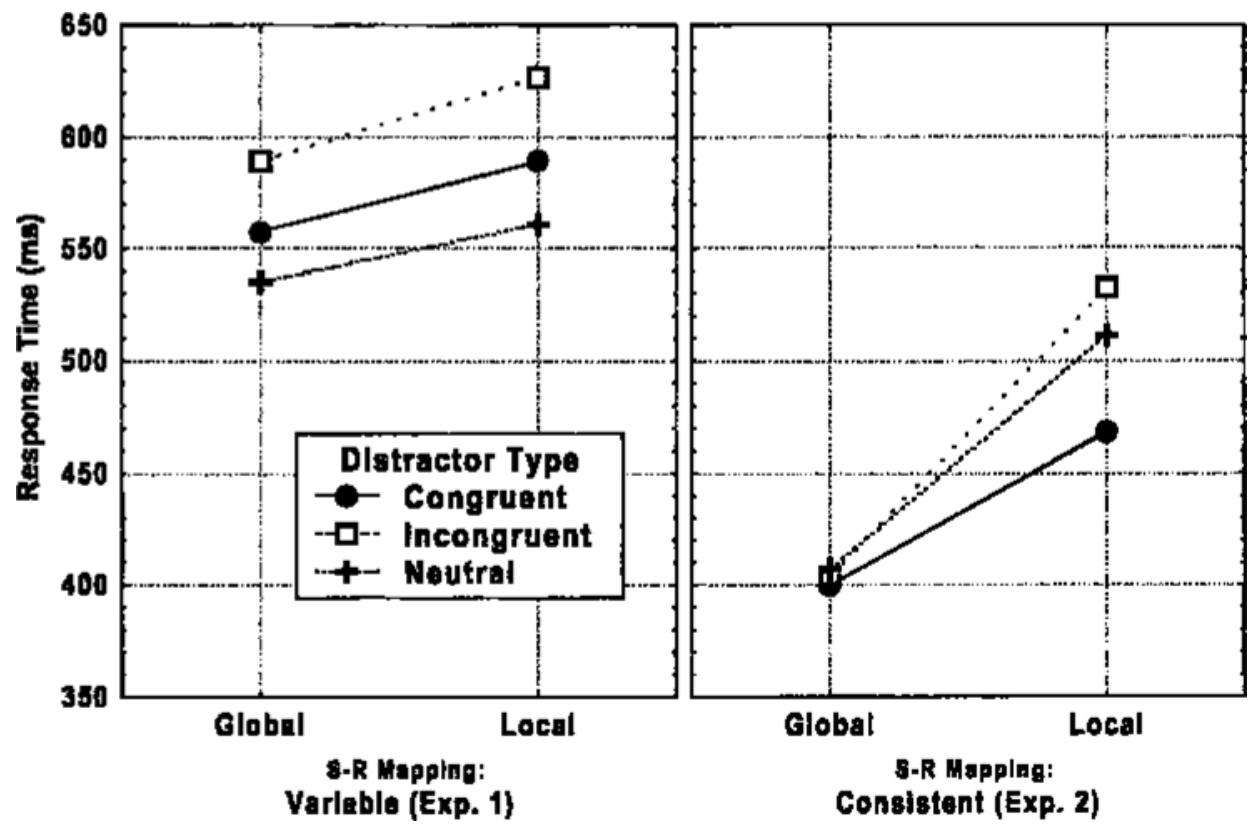

Figure 2. Pattern of results for congruent, incongruent, and neutral distractors, comparing response times in the variable $S-R$ task (Experiment 1; left panel) and the consistent $S-R$ task (Experiment 2; right panel).

tractor type on global responses and on local responses showed one significant difference. Responses to global targets were less accurate when ignored local distractors were incongruent than when they were neutral $(p<.05)$. None of the other comparisons were significant, within global attention conditions or within local attention conditions.

\section{Discussion}

Experiment 1 clearly demonstrated bidirectional interference, strongly indicating that, under these task conditions, the local elements could not be filtered, even under focused attention conditions. Incongruent local distractors interfered with global processing as much as or more than incongruent global distractors interfered with local processing.

Experiment 2 was run to address possible concerns about interpretation of the results. It is important to emphasize that we are talking about a global speed of processing advantage that occurs under specific stimulus conditions. As mentioned above, varying the size (Kinchla $\&$ Wolfe, 1979), local element density (Klein \& Barresi, 1985; Martin, 1979), eccentricity (Grice et al., 1983), stimulus duration (Paquet \& Merikle, 1984), and other stimulus factors will determine whether global or local precedence is observed. Our interest is in the case in which, using the same stimulus set and the same opportunity for selective attention, local interference is not observed for the traditional consistent S-R mapping task and is observed for the variable $\mathrm{S}-\mathrm{R}$ mapping task. We have argued that ignored local items are identified (see also
Briand, 1993) and that our varied S-R mapping contributed to the ability to observe local-to-global interference because of the altered decision process for stimuli that change their role as targets or distractors from trial to trial. We still need to show that varied $\mathrm{S}-\mathrm{R}$ mapping is the critical factor. In other words, we have not yet shown that local-to-global interference is not obtained when we use a consistent $\mathrm{S}-\mathrm{R}$ mapping using our stimuli.

\section{EXPERIMENT 2 Consistent S-R Mapping}

Experiment 2 used the same compound figures presented at the same visual angle and stimulus duration as Experiment 1. The main differences involved the task and the mapping between stimulus and response. As in Experiment 1, attention was directed to either the global level or the local level for the entire block, and the participants were informed prior to the block which level was relevant and which level should be ignored. However, the task was a two-alternative forced-choice task, requiring a response on every trial. There were only two targets: the digit " 1 " and the digit " $2 . "$ These targets were consistently mapped to a left-hand keypress for the digit " 1 " and a right-hand keypress for the digit " 2 ." Thus, on every trial in random order, either a " 1 " or a " 2 " appeared at the attended level (see Figure 3 for examples). In addition, on every trial, the distractor that appeared at the ignored level was congruent (the same digit), incongruent (the opposite digit), or neutral (one of the other seven digits) 

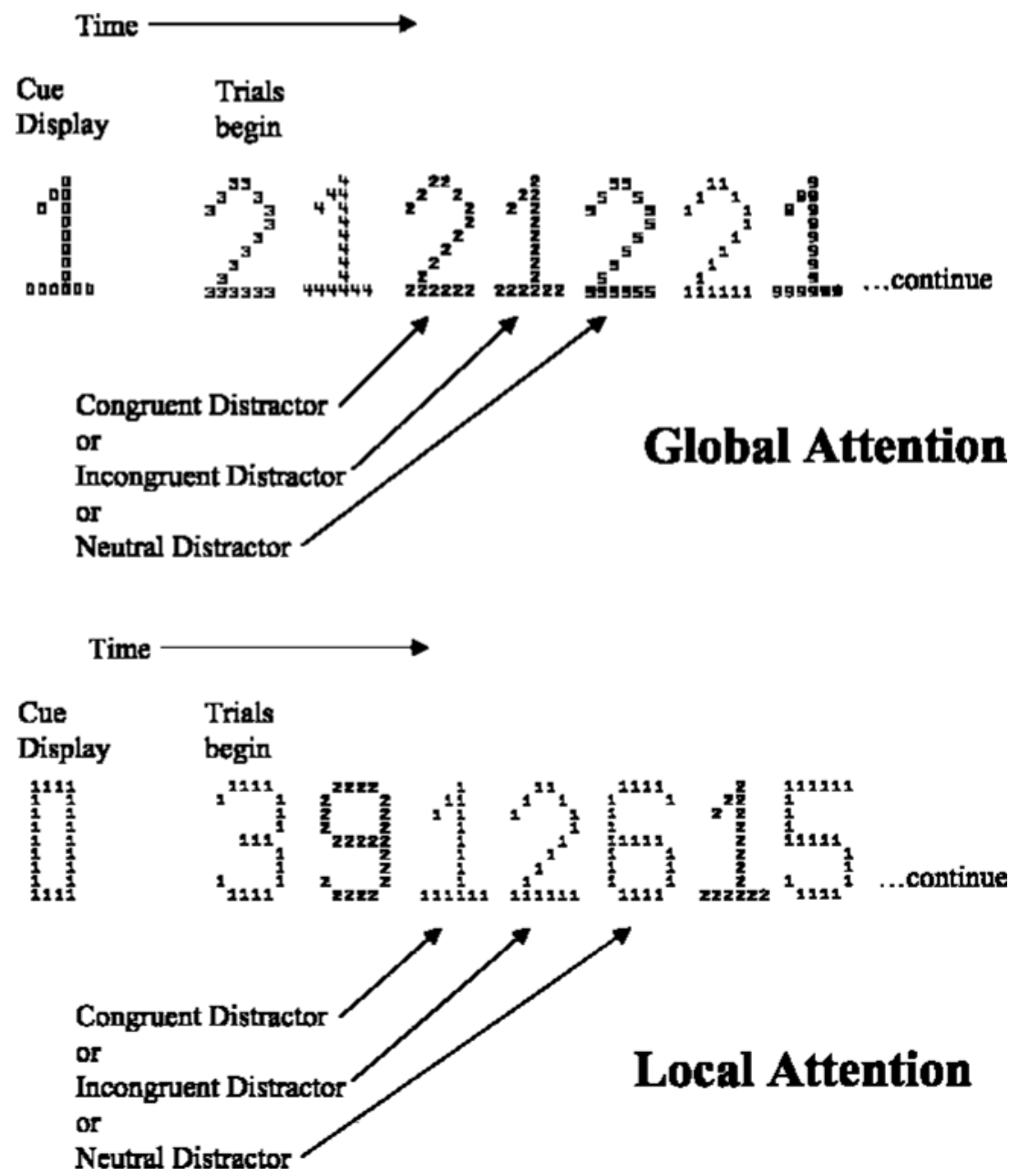

Figure 3. Consistent S-R mapping task. Examples of possible sequences of stimuli in the two-alternative forced-choice task are shown for global attention (upper example) and local attention (lower example). Only the first 7 of 30 trials are shown. The cue display at the beginning of the block of trials informed the participant whether to direct attention globally or locally, and it remained on the screen until the space bar was pressed to start the trials. The onset of the first stimulus occurred after a 1,500-msec delay. Stimulus duration was $100 \mathrm{msec}$, and the screen remained blank until the response was made. The onset of each following stimulus occurred $1,500 \mathrm{msec}$ following the response to the previous stimulus. On each trial, the item at the ignored level was a congruent distractor (the digit at the ignored level was the same as the target digit), an incongruent distractor (the digit at the ignored level was the digit mapped to the opposite response to the target digit), or a neutral distractor (the digit at the ignored level was any one of the other seven digits, which never required a response).

with respect to the required response. There were 30 blocks in which attention was directed locally and 30 blocks in which attention was directed globally.

\section{Method}

Participants. The participants were 10 undergraduate and graduate students at McMaster University who volunteered to participate either for class credit (undergraduates) or for a small remuneration (graduates). All participants had normal or corrected-to-n ormal vision.

Stimuli and Procedure. The hierarchical stimuli were identical to the stimuli used in Experiment 1, and the stimulus duration remained at $100 \mathrm{msec}$. The task, however, was substantially different. There were only two targets: the digits " 1 " and " 2 ." The task was a two-alternative forced-choice design, such that, on each trial, the par- ticipant was required to press one key ("z" on the computer keyboard) with the left index finger in response to the digit " 1 " or to press a different key ("/" on the computer keyboard) with the right index finger in response to the digit " 2 ." This S-R mapping was consistent throughout the experiment, and no other responses were required.

As in Experiment 1, the participants were informed prior to each block whether to attend to the global or local items. The attended level was the same for the duration of the block.

Each session consisted of four practice blocks (two each of local and global attention), followed by 36 test blocks in random order (18 each of local and global attention). The participants were asked to respond as quickly as possible without sacrif icing accuracy.

Each block consisted of 30 trials. Examples of the first 7 trials from a global attention block and a local attention block are shown in Figure 3. Single hierarchical stimuli were presented at fixation. On 
every trial, either a " 1 " or a "2" appeared at the attended level (15 trials each, in random order). Three distractor conditions (congruent / incongruent /neutral) were presented equally often ( 5 trials each for each of the two targets, in random order). A congruent distractor was the same as the target digit ("1" or " 2 "), an incongruent distractor was the digit mapped to the opposite response ("2" if the target was " 1 ," and " 1 " if the target was " 2 "), and a neutral distractor was randomly chosen from the other seven digits (3 through 9), which were never targets.

The participants pressed the space bar to start each block of trials. After a 1,500-msec delay, the first stimulus was briefly presented for a duration of $100 \mathrm{msec}$. Upon offset of the stimulus, the screen remained blank until the response was made. Following the response, a 1,500-msec delay preceded the next stimulus onset. Feedback was provided at the end of each block, including average RT for correct trials and number of correct trials for each of the two targets.

\section{Results}

RTs for Experiment 2 are plotted in the right panel of Figure 2, and means for RT and proportion correct are presented in Table 1.

Response time. The classic global dominance effect was observed in Experiment 2, such that global responses were faster overall, and local responses were affected by global distractor type, but not vice versa. A two-factor repeated measures ANOVA was performed for level (global/ local) $\times$ distractor type (congruent/incongruent/neutral). The main effect of level again showed that responses to global targets were faster than responses to local targets $\left[F(1,9)=79.93, M S_{\mathrm{e}}=1,880, p<.001\right]$. There was a main effect of distractor type $\left[F(2,18)=99.07, M S_{\mathrm{e}}=\right.$ $62, p<.001]$, and there was a significant interaction between the two factors $\left[F(2,18)=55.22, M S_{\mathrm{e}}=82, p<\right.$ $.001]$. A comparison of means was performed to determine the effect of the three types of distractors on global targets and on local targets. Distractor type did not affect global responses (Scheffé $p>$.6; Newman-Keuls $p>$.2). For local responses, however, incongruent global distractors resulted in slower responses than congruent distractors (Scheffé $p<.001)$ and neutral distractors (Scheffé $p<.01$ ), and neutral global distractors resulted in slower responses than congruent distractors (Scheffé $p<01$ ).

Accuracy. Global dominance was also reflected in the accuracy measures, with higher accuracy for global responses than for local responses, and a disadvantage for local targets when the global distractors were incongruent. Applying the same two-factor repeated measures ANOVA, we found two main effects and a significant interaction. Accuracy was greater for global targets than for local targets $\left[F(1,9)=12.13, M S_{\mathrm{e}}<0.001, p<.01\right]$. Distractor type was significant $\left[F(2,18)=17.19, M S_{\mathrm{e}}=\right.$ $0.001, p<.001]$, and the interaction was significant $\left[F(2,18)=9.7, M S_{\mathrm{e}}=0.001, p<.01\right]$. When global distractors were incongruent, responses to local targets were less accurate than responses in all other conditions (Scheffé $p<.01)$. None of the other comparisons were significant (Scheffé $p>9$ ).

\section{Discussion}

The results of Experiment 2 confirmed that our ignored local distractors do not affect responses to global targets when we use a consistent S-R mapping task. The RT means showed a clear global advantage, in terms of faster global responses and unidirectional global-tolocal interference. Furthermore, global responses appeared to be immune to the identity of local distractors in terms of accuracy as well.

In summary of the two experiments, responses to global targets in the variable S-R task were slowed relative to those in the consistent $\mathrm{S}-\mathrm{R}$ task, to a greater degree than were responses to local targets. Importantly, incongruent local distractors affected global RTs only in the variable $\mathrm{S}-\mathrm{R}$ task. In contrast, the RT results from the consistent $\mathrm{S}-\mathrm{R}$ task showed the typical global advantage without showing interference from incongruent local distractors.

These results support the idea that at least part of the reason we were able to observe the bidirectional interference in Experiment 1 was due to the variable mapping between stimulus and response, which required a greater degree of semantic analysis for each stimulus. There are two other differences between the two experiments that might have contributed to increase the processing demands of Experiment 1 relative to Experiment 2. It is possible that the number of potentially relevant stimuli could be a factor (nine in Experiment 1 vs. two in Experiment 2). It is also possible that processing demands might differ between the go/no-go responses and the two-alternative forced-choice responses. In any case, the explanation based on increased processing demands at later stages still holds.

\section{GENERAL DISCUSSION}

To summarize our results, under consistent S-R mapping conditions, it appeared that the identity of the ignored local distractors did not affect global responses, and unilateral global-to-local interference was observed. In contrast, when the variable $\mathrm{S}-\mathrm{R}$ mapping was used, global responses were very sensitive to ignored local information, and bilateral global-to-local and local-to-global interference was observed. The two tasks were not different in terms of directed attention or blocked attention conditions; in both cases, the participants were instructed to select only the global levels or the local levels for an entire block of trials. In addition, distractors on nontarget trials in the variable $\mathrm{S}-\mathrm{R}$ task were incompatible with required no-go responses, so that the participants were highly motivated to selectively attend to the relevant level. Moreover, the stimuli used in both tasks were identical, so the differing patterns of results cannot be explained by differences in perceptual discriminability.

The critical factor seems to be that the tasks differed in the responses that were required. The consistent $\mathrm{S}-\mathrm{R}$ task, which is the norm for most global/local studies, involved a two-alternative forced choice, for which one of the two possible responses was required on every trial. The variable S-R task was a go/no-go task, involving a single response, which was withheld on nontarget trials and executed on target trials. The more dramatic difference between the two tasks was that the participants were able 
to learn a consistent mapping between the two target stimuli and the two possible responses in the consistent S-R task. In contrast, the identity of the targets and distractors changed from trial to trial in the variable S-R task.

Why does bidirectional interference occur in the variable $\mathrm{S}-\mathrm{R}$ mapping task and not in the consistent $\mathrm{S}-\mathrm{R}$ mapping task? This question relates directly to the source of the global advantage. Some attempts to account for the source of the global advantage have supported an early perceptual source (Hughes et al., 1990; Hughes et al., 1984; Hughes et al., 1996; May, Gutierrez, \& Harsin, 1995; Shulman \& Wilson, 1987), and others have supported the idea that the source is postperceptual (Boer \& Keuss, 1982; Delis, Robertson, \& Efron, 1986; Lamb \& Robertson, 1989; Miller, 1981; Robertson, Lamb, \& Knight, 1988; Ward, 1982). A review of many of these studies can be found in Kimchi (1992). In favor of the idea that the global advantage is a perceptual effect, there is evidence for a strong relation between global and local information processing and low and high spatial frequencies, respectively (Shulman, Sullivan, Gish, \& Sakoda, 1986; Shulman \& Wilson, 1987). The global advantage may be accounted for by differences in transmission rates for low spatial frequency information (carried by the magnocellular pathway) and high spatial frequency information (carried by the parvocellular pathway; De Valois \& De Valois, 1988). Low spatial frequencies, which usually carry the global information, are available earlier than are higher spatial frequencies (Breitmeyer, 1975; De Valois \& De Valois, 1988; Hughes et al., 1996). The magnocellular pathway has faster conduction rates, there is a higher contrast gain, and there may even be inhibition between parallel spatial frequency channels that further enhances the dominance of global information (Hughes et al., 1996; May et al., 1995). This does not preclude the argument that local information and global information accumulate at the same time; it simply suggests that global information accumulates faster at early stages.

Several studies have shown that the global RT advantage is eliminated or greatly reduced if the low spatial frequencies in the compound stimuli are eliminated by presenting highpass-filtered stimuli or by using contrastbalanced stimuli (Hughes et al., 1990; Lamb \& Yund, 1993; Lamb, Yund, \& Pond, 1999; Michimata, Okubo, \& Mugishima, 1999). Such evidence supports the hypothesis that the global advantage has an early perceptual source. If global precedence arises at least in part from the more efficient processing of lower spatial frequencies in the magnocellular stream, this could subsequently result in greater salience and attention-grabbing power because of faster conduction of information. These two factors together provide the global advantage. Evidence from brain-imaging studies supports the hypothesis that attention enhances processing of global information, relative to local information, as early as extrastriate cortical areas (Evans et al., 2000; Fink et al., 1996; Fink et al., 1997). An important consideration for our arguments is that this advantage at early stages may be measured at the response stage under conditions for which fast responses can be made. If evaluation of the stimulus must become more deliberate, global processing may lose its advantage over local processing.

Consistent S-R mapping may facilitate the development of fast, automatic responses (Schneider \& Shiffrin, 1977). Because the varied S-R mapping induces a greater degree of semantic analysis, the time required to respond to global targets is increased. In that case, the initial global speed advantage may contribute less to the overall processing time, resulting in more equal global and local RTs. In other words, in our variable S-R task, there may be some "catch-up" involved for the local information. Even though attention is directed to either global or local elements for the duration of a block of trials, if ignored items are processed along with attended items, then a higher level of semantic analysis will occur for the ignored items as well. In fact, relative to consistent $S-R$ mapping tasks, our results showed that, under varied mapping conditions, responses to global targets were slowed to a greater degree than were responses to local targets. This should allow ignored local information to have a greater effect on responses, resulting in bidirectional interference.

Why does our manipulation affect global responses to a greater extent than local responses? Hübner (1997) hypothesized that, under conditions in which fast processing of information at a level is not possible, there is a decrease in the contribution of low spatial frequencies to the global advantage. This makes sense if one considers that the speed advantage occurs at the very early stages. Once later stages are reached, processing for local and global most likely continues at similar rates. If the task becomes demanding at later stages, the local processing can catch up to a point at which it interferes with global responses. In our case, fast processing was made difficult by requiring semantic analysis on every trial. What we have shown is that, under the same focused attention conditions that show a clear global dominance when responses are fast, responses can be slowed and global dominance can be eliminated by increasing the processing demands at later stages of semantic evaluation. This slowing of responses was much more dramatic for global targets than for local targets, suggesting that there were new influences on global processing (namely, the interference from local elements). Local processing was also slowed but not to the same extent because interference from global elements was not a new influence (global elements interfere with local in any case). Thus, the biggest change was for global processing.

There is another factor in our variable $S-R$ mapping design and the number-sequence task that may be important to understanding the nature of the interference. In our task, location information must be confirmed. In global/local tasks with consistent S-R mapping, participants have a target (or targets) in mind and search each display, making particular responses when the expected item matches the presented item. In other words, a match is coupled with a response. In the number-sequence task, 
however, a target is defined by a nonmatch rather than a match. The target for the response is different from the target for the match on each trial. In the incongruent distractor condition, the expected digit appears at the ignored level. If the ignored item is identified, then evidence for both a nonmatch (with the target) and a match (with the distractor) occurs. In that case, there may be some confusion about the locations from which the match and nonmatch information arose. Given that information about both global and local identity is available, the slowed responses that occur in the incongruent condition may be due in part to extra processing required to confirm the location of the incongruent item. Clearly, this is a very late process, occurring for confirmatory purposes after all items have been identified.

If we accept that global information and local information are available together at early stages of processing (Boer \& Keuss, 1982; Evans et al., 2000; Miller, 1981) and that global information and local information are accumulating in support of identification of both global and local elements, then it makes some sense that it might be efficient to perform the identification in separate processing streams. When identity of both local and global is needed, it would not be efficient for the information to accumulate toward the same identifier. There are many studies that support a right- and left-hemisphere bias for processing global and local aspects of a stimulus, respectively. This evidence comes from behavioral studies (Blanca, Zalabardo, Garcia-Criado, \& Siles, 1994; Kimchi \& Merhav, 1991; Shedden, Marsman, \& Paul, 2001), experiments in neuropsychology (Delis et al., 1986; Lamb, Robertson, \& Knight, 1989; Robertson \& Delis, 1986; Robertson \& Lamb, 1991; Robertson, Lamb, \& Zaidel, 1993), and brain-imaging studies (Evans et al., 2000; Fink et al., 1996; Heinze, Hinrichs, Scholz, Burchert, \& Mangun, 1998; Heinze \& Munte, 1993). One advantage of parallel streams in the right and left hemispheres is the ability to process in parallel different kinds of information that must be integrated yet remain separate at the same time. We do not lose the individuation of the parts when we contemplate the whole.

At early stages of processing, the visual system constructs a detailed image from relatively simple features (Hubel \& Wiesel, 1959; Hubel, Wiesel, \& Stryker, 1977; Livingstone \& Hubel, 1987). The specificity of responses of cells in early visual areas and the increasing complexity of effective stimuli as one travels up the visual processing stream suggest that it is not unreasonable to hypothesize that recognition of an object must in some sense begin with an analysis of its parts from which the whole is constructed. Simple features exist for both global and local elements, although at different spatial frequencies, and these simple features are analyzed at the same time at early visual stages. The lower spatial frequencies inherent in the global information may be processed more quickly than the higher spatial frequencies of the local information, leading to faster accumulation of global information at early stages. This advantage is enhanced with global attention, and a strong global advantage is observed when fast responses are possible. However, analysis of local information does not wait for some aspect of global analysis to complete, but it occurs along with global analysis, even when local elements are not attended. When fast responses are not possible, speed of processing at early stages contributes less to the overall RT, and global responses become as sensitive to local information as local responses are to global information.

\section{REFERENCES}

Blanca, M. J., Zalabardo, C., Garcia-Criado, F., \& Siles, R. (1994). Hemispheric differences in global and local processing dependent on exposure duration. Neuropsychologia, 32, 1343-1351.

Boer, L. C., \& Keuss, P. J. G. (1982). Global precedence as a postperceptual effect: An analysis of speed-accuracy tradeoff functions. Perception \& Psychophysics, 31, 358-366.

Breitmeyer, B. G. (1975). Simple reaction time as a measure of the temporal response properties of transient and sustained channels. $\mathrm{Vi}$ sion Research, 15, 1411-1412.

BRIAND, K. A. (1993). Efficient filtering of irrelevant global and local information when target level and location are random. Psychological Research, 55, 264-269.

BRIAND, K. A. (1994). Selective attention to global and local structure of objects: Alternative measures of nontarget processing. Perception \& Psychophysics, 55, 562-574.

Delis, D. C., Robertson, L. C., \& Efron, R. (1986). Hemispheric specialization of memory for visual hierarchical stimuli. Neuropsychologia, 24, 205-214.

De Valois, R. L., \& De Valois, K. K. (1988). Spatialvision. New York: Oxford University Press.

DunCAN, J. (1981). Directing attention in the visual field. Perception \& Psychophysics, 30, 90-93.

Evans, M. A., Shedden, J. M., Hevenor, S. J., \& Hahn, M. C. (2000). Parallel processing of local and global aspects of hierarchical stimuli: Evidence for lateralization at early stages of processing. Neuropsychologia, 38, 225-239.

Fink, G. R., Halligan, P. W., Marshall, J. C., Frith, C. D., FrackOWIAK, R. S. J., \& Dolan, R. J. (1996). Where in the brain does visual attention select the forest and the trees? Nature, 382, 626-628.

Fink, G. R., Marshall, J. C., Halligan, P. W., Frith, C. D., FrackOWIAK, R. S. J., \& Dolan, R. J. (1997). Hemispheric specialization for global and local processing: The effect of stimulus category. Proceedings of the Royal Society of London, 264, 487-494.

Grice, G. R., Canham, L., \& Boroughs, J. M. (1983). Forest before trees? It depends where you look. Perception \& Psychophysics, 33, 121-128.

Heinze, H.-J., Hinrichs, H., Scholz, M., Burchert, W., \& Mangun, G. R. (1998). Neural mechanisms of global and local processing: A combined PET and ERP study. Journal of Cognitive Neuroscience, 10, 485-498.

Heinze, H.-J., \& MunTe, T. F. (1993). Electrophysiological correlates of hierarchical stimulus processing: Dissociation between onset and later stages of global and local target processing. Neuropsychologia, 31, 841-852.

HoEgER, R. (1997). Speed of processing and stimulus complexity in lowfrequency and high-frequency channels. Perception, 26, 1039-1045.

Hoffman, J. E. (1980). Interaction between global and local levels of a form. Journal of Experimental Psychology: Human Perception \& Performance, 6, 222-234.

Hubel, D. H., \& Wiesel, T. N. (1959). Receptive fields of single neurones in the cat's striate cortex. Journal of Physiology, 148, 574-591.

Hubel, D. H., Wiesel, T. N., \& Stryker, M. P. (1977). Orientation columns in macaque monkey visual cortex demonstrated by the 2-deoxyglucose autoradiographic technique. Nature, 269, 328-330. 
HÜBNER, R. (1997). The effect of spatial frequency on global precedence and hemispheric differences. Perception \& Psychophysics, 59, 187-201.

Hughes, H. C., Fendrich, R., \& Reuter-Lorenz, P. A. (1990). Global versus local processing in the absence of low spatial frequencies. Journal of Cognitive Neuroscience, 2, 272-282.

Hughes, H. C., Layton, W. M., Baird, J. C., \& Lester, L. S. (1984). Global precedence in visual pattern recognition. Perception \& Psychophysics, 35, 361-371.

Hughes, H. C., Nozawa, G., \& Kitterle, F. (1996). Global precedence, spatial frequency channels, and the statistics of natural images. Journal of Cognitive Neuroscience, 8, 197-230.

KIMCHI, R. (1992). Primacy of wholistic processing and global/local paradigm: A critical review. Psychological Bulletin, 112, 24-38.

Kimchi, R, \& MerhaV, I. (1991). Hemispheric processing of global form, local form, and texture. Acta Psychologica, 76, 133-147.

Kinchla, R. A., \& Wolfe, J. M. (1979). The order of visual processing: "Top-down," "bottom-up," or “middle-out." Perception \& Psychophysics, 25, 225-231.

Klein, R. M., \& BArresi, J. (1985). Perceptual salience of form versus material as a function of variations in spacing and number of elements. Perception \& Psychophysics, 37, 440-446.

LAMB, M. R, \& RoberTSON, L. C. (1988). The processing of hierarchical stimuli: Effects of retinal locus, locational uncertainty, and stimulus identity. Perception \& Psychophysics, 44, 172-181.

LAMb, M. R., \& RoberTson, L. C. (1989). Do response time advantage and interference reflect the order of processing of global- and locallevel information? Perception \& Psychophysics, 46, 254-258.

LAMB, M. R., \& RoBERTSON, L. C. (1990). The effect of visual angle on global and local reaction times depends on the set of visual angles presented. Perception \& Psychophysics, 47, 489-496.

Lamb, M. R, Robertson, L. C., \& KNight, R. T. (1989). Attention and interference in the processing of global and local information: Effects of unilateral temporal-parietal junction lesions. Neuropsychologia, 27, 471-483.

LAMb, M. R., \& YUnd, E. W. (1993). The role of spatial frequency in the processing of hierarchically organized stimuli. Perception \& Psychophysics, 54, 773-784.

Lamb, M. R., Yund, E. W., \& Pond, H. M. (1999). Is attentional selection to different levels of hierarchical structure based on spatial frequency? Journal of Experimental Psychology: General, 128, 88-94.

Livingstone, M. S., \& Hubel, D. H. (1987). Psychophysical evidence for separate channels for the perception of form, color, movement, and depth. Journal of Neuroscience, 7, 3416-3468.

Logan, G. D., \& Stadler, M. A. (1991). Mechanisms of performance improvement in consistent mapping memory search: Automaticity or strategy shift? Journal of Experimental Psychology: Learning, Memory, \& Cognition, 17, 478-496.

MARTIN, M. (1979). Local and global processing: The role of sparsity. Memory \& Cognition, 7, 476-484.

May, J. G., Gutierrez, C., \& Harsin, C. A. (1995). The time-course of global precedence and consistency effects. International Journal of Neuroscience, 80, 237-245.

Melara, R. D., \& Mounts, J. R. W. (1993). Selective attention to Stroop dimensions: Effects of baseline discriminability, response mode, and practice. Memory \& Cognition, 21, 627-645.

Michimata, C., OKubo, M., \& Mugishima, Y. (1999). Effects of background color on the global and local processing of hierarchically organized stimuli. Journal of Cognitive Neuroscience, 11, 1-8.

Miller, J. (1981). Global precedence in attention and decision. Jour- nal of Experimental Psychology: Human Perception \& Performance, 7, 1161-1174.

NAVON, D. (1977). Forest before trees: The precedence of global features in visual perception. Cognitive Psychology, 9, 353-383.

Navon, D. (1981). The forest revisited: More on global precedence. Psychological Research, 43, 1-32.

PAQUeT, L. (1992). Global and local processing in nonattended objects: A failure to induce local processing dominance. Journal of Experimental Psychology: Human Perception \& Performance, 18, 512-529.

Paquet, L., \& Merikle, P. M. (1984). Global precedence: The effect of exposure duration. Canadian Journal of Psychology, 38, 45-53.

Paquet, L., \& Merikle, P. M. (1988). Global precedence in attended and nonattended objects. Journal of Experimental Psychology: Human Perception \& Performance, 14, 89-100.

Parker, D. M., Lishman, J. R., \& Hughes, J. (1996). Role of coarse and fine spatial information in face and object processing. Journal of Experimental Psychology: Human Perception \& Performance, 22, $1448-1466$.

Pomerantz, J. R. (1983). Global and local precedence: Selective attention in form and motion perception. Journal of Experimental Psychology: General, 112, 516-540.

Robertson, L. C., \& Delis, D. C. (1986). "Part-whole" processing in unilateral brain-damaged patients: Dysfunction of hierarchical organization. Neuropsychologia, 24, 363-370.

Robertson, L. C., \& LAMB, M. R. (1991). Neuropsychological contribution to theories of part/whole organization. Cognitive Psychology, 23, 299-330.

Robertson, L. C., Lamb, M. R., \& Knight, R. T. (1988). Effects of lesions of temporal-parietal junction on perception and attention processing in humans. Journal of Neuroscience, 8, 3757-3769.

Robertson, L. C., LAMB, M. R., \& ZAIDEL, E. (1993). Interhemispheric relations in processing hierarchical patterns: Evidence from normal and commissurotomized patients. Neuropsychology, 7, 325-342.

Schneider, W., \& Shiffrin, R. M. (1977). Controlled and automatic human information processing: I. Detection, search, and attention. Psychological Review, 84, 1-66.

Shedden, J. M., Marsman, I. A., \& Paul, M. P. (2001). Global/local attention switching: Effects of hemisphere bias and level repetition. Manuscript submitted for publication.

Shiffrin, R. M., \& Schneider, W. (1977). Controlled and automatic human information processing: II. Perceptual learning, automatic attending, and a general theory. Psychological Review, 84, 127-190.

Shulman, G. L., Sullivan, M. A., Gish, K., \& SaKoda, W. J. (1986). The role of spatial-frequency channels in the perception of local and global structure. Perception, 15, 259-273.

Shulman, G. L., \& WiLson, J. (1987). Spatial frequency and selective attention to local and global information. Perception, 16, 89-101.

VAN DER HEIJDEN, A. H. C. (1981). Short-term visual information forgetting. London: Routledge \& Kegan Paul.

Van der Heijden, A. H. C., HagenaAr, R., \& Bloem, W. (1984). Two stages in postcategorical filtering and selection. Memory \& Cognition, 12, 458-469.

WARD, L. M. (1982). Determinants of attention of local and global features of visual forms. Journal of Experimental Psychology: Human Perception \& Performance, 8, 562-581.

(Manuscript received December 9, 1998; revision accepted for publication June 7, 2000.) 